\title{
S100A2 Silencing Relieves Epithelial-Mesenchymal Transition in Pulmonary Fibrosis by Inhibiting the Wnt/ $\beta$-Catenin Signaling Pathway
}

\author{
Guichuan Huang,, Jing Zhang, ${ }^{2}$ Gang Qing,, Daishun Liu, ${ }^{3}$ Xin Wang, \\ Yi Chen, ${ }^{1}$ Yishi Li, and Shuliang Guo ${ }^{1}$
}

Pulmonary fibrosis (PF) is a progressive and lethal disease with poor prognosis. S100A2 plays an important role in the progression of cancer. However, the role of $S 100 A 2$ in PF has not yet been reported. In this study, we explored the potential role of $S 100 A 2$ in PF and its potential molecular mechanisms. Increased expression of S100A2 was first observed in lung tissues of PF patients. We found that downregulation of S100A2 inhibited the transforming growth factor- $\beta 1$ (TGF- $\beta 1$ )-induced epithelial-mesenchymal transition (EMT) in A549 cells. Mechanically, TGF- $\beta 1$ upregulated $\beta$-catenin and the phosphorylation of glycogen synthase kinase- $3 \beta$, which was blocked by silencing S100A2 in vitro. Furthermore, lithium chloride (activator of the Wnt/ $\beta$-catenin signaling pathway) effectively rescued S100A2 knockdown-mediated inhibition of EMT in PF. In conclusion, these findings demonstrate that downregulation of S100A2 alleviated PF through inhibiting EMT. S100A2 is a promising potential target for further understanding the mechanism and developing a strategy for the treatment of PF and other EMT-associated diseases.

Keywords: pulmonary fibrosis, epithelial-mesenchymal transition, $S 100 A 2$, transforming growth factor- $\beta 1$

\section{Introduction}

$\mathbf{P}$ ULMONARY FIBROSIS (PF) is a common outcome of various interstitial lung diseases, including coronavirus disease-2019 (COVID-19), pneumoconiosis, drug-induced fibrosis, and idiopathic PF (Du et al., 2019; George et al., 2020; He et al., 2020). PF is characterized by alveolar epithelial cell (AEC) injury, and the excessive proliferation of mesenchymal cells in the interstitium, which leads to the exaggerated accumulation of extracellular matrix (ECM) and distorted lung architecture. Although we have made great progress in the diagnosis of and treatment for PF in recent years, the survival rate has not improved (Kumar et al., 2018). Therefore, it is crucial to explore the precise molecular mechanisms involved in the development of PF and to determine targets for the diagnosis and treatment of PF.

Epithelial-mesenchymal transition (EMT) is a process in which epithelial cells are transformed into a mesenchymal phenotype, with the loss of contact adhesion and apical-basal polarity, acquisition of the mesenchymal property of invasion, migration, and production of ECM (Jolly et al., 2018). An increasing number of studies have shown that EMT plays an important role in the pathogenesis of PF (Salton et al., 2019). In addition, transforming growth factor- $\beta 1$ (TGF- $\beta 1$ ) is a key profibrotic factor that has been implicated to induce EMT during PF (Willis and Borok, 2007).

The S100 protein family is a group of highly conserved elongation factor (EF)-hand calcium-binding proteins (Chen et al., 2014). S100A2 is an important member of the S100 protein family and has been involved in various cancers (Wolf et al., 2011). Naz et al. (2014) reported that S100A2 promoted the occurrence of cancer through regulating PI3K/Akt signaling and the functional interaction with Smad3. In addition, a study indicated that the p53S100A2 positive feedback loop negatively regulates epithelialization in cutaneous wound healing (Pan et al., 2018). However, the role of S100A2 in fibrosis, including PF, has not been investigated.

In this study, we demonstrated that $100 A 2$ was increased in lung tissues of patients with PF. Downregulation of S100A2 inhibited TGF- $\beta 1$-induced EMT in human type II AECs through blocking the $\mathrm{Wnt} / \beta$-catenin pathway in vitro. These results will increase our understanding of the pathological mechanisms of PF.

\footnotetext{
${ }^{1}$ Department of Pulmonary and Critical Care Medicine, The First Affiliated Hospital of Chongqing Medical University, Chongqing, China.

${ }^{2}$ Department of Pulmonary and Critical Care Medicine, Affiliated Hospital of Zunyi Medical University, Zunyi, China.

${ }^{3}$ Zunyi Medical University, Zunyi, China.
} 


\section{Materials and Methods}

\section{Ethics statement}

The study was approved by the ethics committee of The First Affiliated Hospital of Chongqing Medical University (No. 2020-147), and the study was in accordance with the Declaration of Helsinki.

\section{Data collection from the Gene Expression Omnibus database}

We searched microarray data of S100A2 in the Gene Expression Omnibus (GEO) database. The following keywords were used: (lung fibrosis OR pulmonary fibrosis). The search results were further restricted as following: Series [Entry type] and Homo sapiens [Organism]. Inclusion criteria for microarrays were as follows: (1) the data set included fibrotic and nonfibrotic tissues; (2) the number of samples in fibrotic and nonfibrotic tissues was at least two; (3) data set provided the expression values of $S 100 A 2$ in fibrotic and nonfibrotic tissues.

\section{Tissues, cell line, and cell culture}

Twelve PF tissues were obtained from patients undergoing lung biopsy. A total of 17 control lung tissues were collected from the normal areas of the peripheral lung removed during lung cancer resection. All tissues were obtained from the First Affiliated Hospital of Chongqing Medical University.

The human type II AEC line, A549, was purchased from the Cell Bank of the Chinese Academy of Sciences. Cells were cultured in Roswell Park Memorial Institute-1640 (RPMI-1640) medium supplemented with $10 \%$ fetal calf serum (PAN), $100 \mathrm{U} / \mathrm{mL}$ penicillin, and $100 \mu \mathrm{g} / \mathrm{mL}$ streptomycin (Gibco) at $37^{\circ} \mathrm{C}$ in a $5 \%$ carbon dioxide atmosphere. A549 cells were treated with recombinant human TGF- $\beta 1$ (Peprotech, NJ) at final concentration of $10 \mathrm{ng} / \mathrm{mL}$ for $48 \mathrm{~h}$ with or without $20 \mathrm{mmol} / \mathrm{L}$ lithium chloride $(\mathrm{LiCl}$; an agonist of the Wnt/ $\beta$-catenin signaling pathway) (Sigma, USA). Then, the cells were collected for further analysis.

\section{Lentivirus transfection}

To establish stable genetic silencing of S100A2, lentivirus was utilized as a vector to carry the interference sequence. The lentivirus vectors inserted with the target gene or a negative control were constructed by Hanbio Biotechnology Company (Shanghai, China). Then, lentivirus was added to $2 \mathrm{~mL}$ complete medium supplemented with a final concentration of $5 \mu \mathrm{g} / \mathrm{mL}$ Polybrene. After $24 \mathrm{~h}$, the medium was replaced with fresh complete medium without lentivirus and Polybrene to culture for another $48 \mathrm{~h}$. Total RNA and protein were collected to confirm the transfection efficiency using quantitative real-time PCR (qRT-PCR) and Western blot.

\section{Quantitative real-time $P C R$}

Total RNA was extracted from the lung tissues or A549 cells using TRIzol reagent (Invitrogen, Carlsbad, CA). The concentration of RNA was measured with the use of the NanoDrop 2000 spectrophotometer (Thermo Scientific, Waltham, MA). Then, $1000 \mathrm{ng}$ of total RNA was reverse transcribed using the PrimeScript RT reagent kit (TaKaRa, Dalian, China). Subsequently, cDNA was amplified by
SYBR Premix Ex Taq (TaKaRa). The relative expression levels of mRNA were normalized to the levels of GAPDH and calculated by the $2^{-\triangle \triangle \mathrm{CT}}$ method. The primers used in this study are listed as follows: $\alpha$-smooth muscle actin $(\alpha$ SMA), forward, 5'-ATGCTCCCAGGGCTGTTTTC-3', reverse, 5'-CTTTTGCTCTGTGCTTCGTC-3'; S100A2, forward, 5'-ACCGACCC TGAAGCAGAACTC-3', reverse, 5'-CCTCA TCTCCCAGCACTCCA-3'; E-cadherin, forward, 5'-CGATTC AAAGTGGGCACAGATG-3', reverse, 5'-GTAGG TGGAGT CCCAGGCGTAG-3'; vimentin, forward, 5'-TCTGGATTCAC TCCCTCT GGTT-3', reverse, 5'-ATCGTGATGCTGAGAAG TTTCGT-3', and GAPDH, forward, 5'-CTTTGGTA TCGTGG AAGGACTC-3', reverse, 5'-GTAGAGGCAGG GATGATG TTCT-3'.

\section{Enzyme-linked immunosorbent assay}

The concentration of E-cadherin, vimentin, and $\alpha$-SMA in the culture medium without dilution was measured using an enzyme-linked immunosorbent assay Kit (Jingmei Biotech., China) according to the manufacturer's protocol.

\section{Hematoxylin and eosin staining}

For hematoxylin and eosin (H\&E) staining analysis, $5 \mu \mathrm{m}$ thick sections of lung tissue were deparaffinized in xylene followed by subjecting to rehydration using an ethanol gradient. The slices were stained with hematoxylin for several minutes. The $70 \%$ and $90 \%$ ethanol were used to dehydrate. Finally, the slices were stained with eosin for few minutes and observed under an Olympus microscope.

\section{Immunohistochemistry}

For immunohistochemistry analysis, $5 \mu \mathrm{m}$ thick sections of lung tissue were deparaffinized in xylene followed by subjecting to rehydration using an ethanol gradient. Next, the endogenous peroxide activity was blocked using $3 \%$ hydrogen peroxide. After blocking with 5\% BSA, the sections were incubated with primary anti-S100A2 (1:400; Abcam) antibodies overnight at $4^{\circ} \mathrm{C}$. Subsequently, the sections were incubated with horseradish peroxidase-conjugated goat antirabbit IgG (Abcam) for $1 \mathrm{~h}$ at room temperature. The sections were developed with the use of diaminobenzidine. The sections were visualized for immunopositivity and images were captured using an Olympus microscope.

\section{Western blot}

Cells were lysed in radioimmunoprecipitation assay lysis buffer containing protease inhibitor cocktail, $1 \%$ phenylmethanesulfonyl fluoride, and $1 \%$ phosphatase inhibitor. Protein concentration was determined with a bicinchoninic acid kit (Beyotime, Biotechnology, China). Equal amounts of protein were separated with $10 \%$ sodium dodecyl sulfate-polyacrylamide gel electrophoresis (SDS-PAGE) gel and transferred to polyvinylidene fluoride membranes. After blocking in 5\% nonfat milk for $2 \mathrm{~h}$, the membranes were incubated with primary antibodies overnight at $4^{\circ} \mathrm{C}$. Then, the membranes were incubated in tris-buffered saline with tween 20 (TBST) supplemented with secondary antibody for $1 \mathrm{~h}$ at room temperature. In this experiment, the following antibodies were used: anti-p-glycogen synthase kinase-3 $\beta$ (p-GSK3 $\beta$ ) (1:1000; Affinity Biosciences), anti-GSK3 $\beta$ (1:500; Affinity Biosciences), 
anti-E-cadherin (1:10,000; Abcam), anti-vimentin (1:3000; Abcam), anti- $\alpha$-SMA (1:2000; Abcam), anti-S100A2 (1:5000; Abcam), anti-GAPDH (1:10,000; Abcam), and anti- $\beta$-catenin (1:5000; Abcam).

\section{Statistical Analysis}

Statistical analyses were performed with SPSS 24.0. All data are expressed as the mean \pm standard deviation. The data were analyzed using independent samples Student's $t$-test between two groups and one-way analysis of variance for more groups with Dunnett's or least significant difference (LSD) post hoc test.

We carried out a comprehensive meta-analysis using STATA 12.0. Continuous outcomes are presented as the standard mean difference (SMD) with $95 \%$ confidence interval $(\mathrm{CI})$. The chi-squared test and $I^{2}$ were used to evaluate the heterogeneity among the studies. A random-effect model was applied if there was heterogeneity among the studies
$\left(I^{2}>50 \%\right.$ or $\left.p_{\text {heterogeneity }}<0.05\right)$. On the contrary, if $I^{2}<50 \%$ or $p_{\text {heterogeneity }}>0.05$, a fixed-effect model was employed. A value of $p<0.05$ was considered statistically significant.

\section{Results}

\section{S100A2 expression was increased in PF obtained from $G E O$ database}

A total of 14 GEO series (GSE) from National Center for Biotechnology Information's (NCBI's) GEO database met the inclusion criteria. However, only eight GSE data sets provided the $S 100 A 2$ expression data. As shown in Supplementary Table $\mathrm{S} 1$, the detailed information for S100A2 expression data from the PF and control groups was extracted based on the GEO database. A total of 168 patients with PF were included. As shown in Figure 1, compared with the control groups, PF groups had a significantly higher expression level of $S 100 A 2$ in accession
A

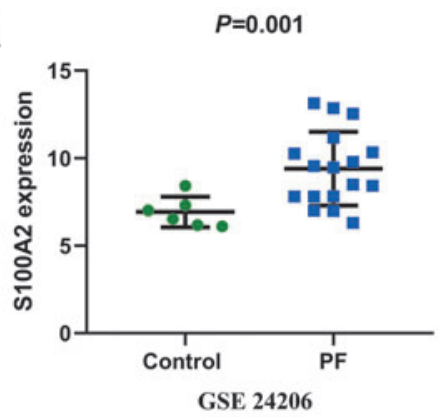

D

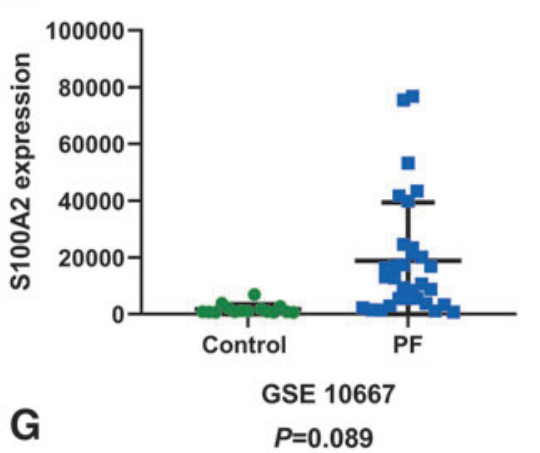

G

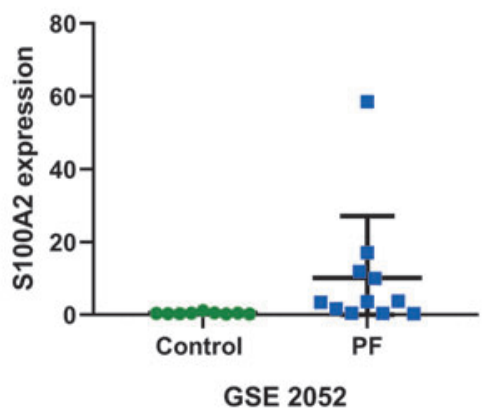

B

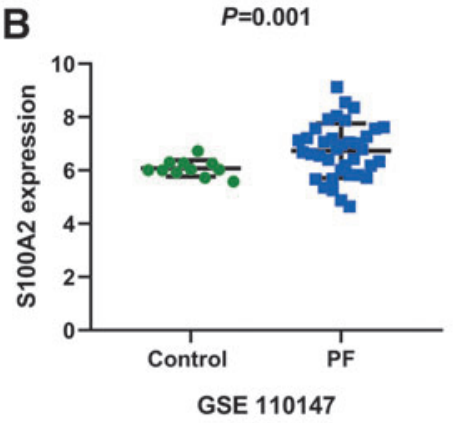

E

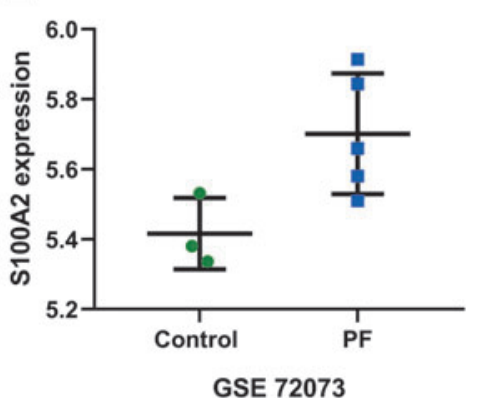

H

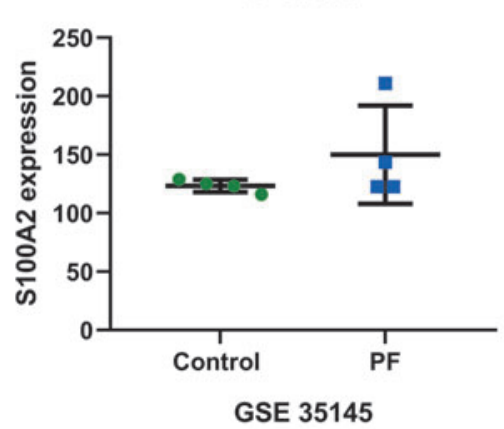

C $P<0.0001$

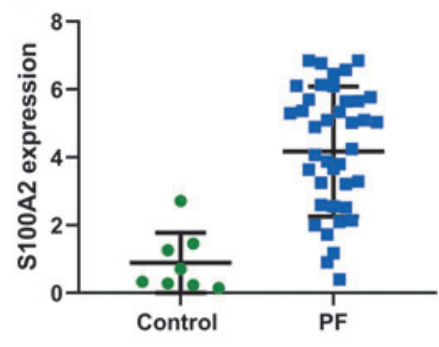

GSE 53845
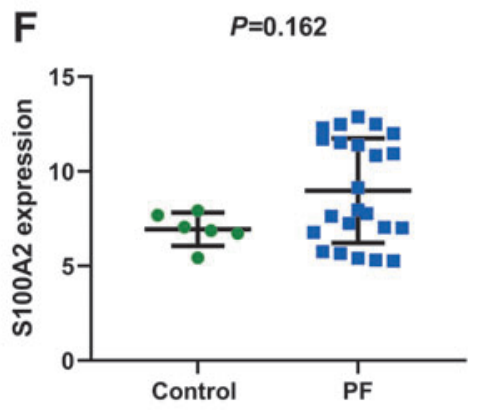

GSE 21369
I

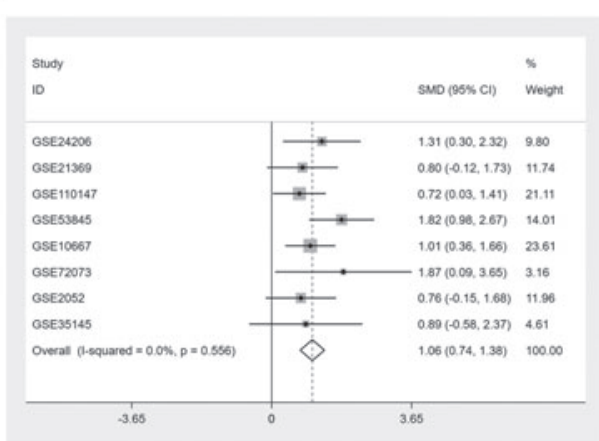

FIG. 1. Expression levels of $S 100 A 2$ in PF and control lung tissues from the GEO database. (A) GSE24206, (B) GSE110147, (C), GSE53845, (D), GSE10667, (E), GSE72073, (F), GSE21369, (G), GSE2052, (H), GSE35145, and (I) meta-analysis for all data sets. CI, confidence interval; GEO, Gene Expression Omnibus; PF, pulmonary fibrosis. 
numbers GSE24206, GSE110147, GSE53845, GSE10667, and GSE72073. Although no statistical differences were observed in the S100A2 expression level between the PF groups and control groups in GSE21369, GSE2052, and GSE35145 (Fig. 1F-H), we found that the PF groups also exhibited higher $S 100 A 2$ expression than the control groups.

Different results existed regarding S100A2 expression in $\mathrm{PF}$ based on the GEO database. Thus, we conducted a metaanalysis using the data for the $S 100 A 2$ expression level from the GEO database. Owing to $I^{2}=0.0 \%$ and $p_{\text {heterogeneity }}>0.05$, a fixed model was applied. As shown in Figure 1I, the combined results revealed that the expression of $S 100 A 2$ was significantly higher in the $\mathrm{PF}$ group than in the control group $(\mathrm{SMD}=1.06,95 \%$ CI: $0.74-1.38, p<0.001)$.

\section{Validation of differential S100A2 expression in PF} in humans both in vivo and in vitro

To further confirm the differential expression of S100A2 during lung fibrosis, we compared its expression in lung fibrosis tissues from PF patients $(n=12)$ with the control $(n=17)$. The detailed information for PF patients is listed in Supplementary Table S2.

The $\mathrm{H} \& \mathrm{E}$ staining was used to assess histopathological changes of lung tissue. As shown in Figure $2 \mathrm{~A}$ and $\mathrm{B}$, the control groups showed that clear alveolar space structure and alveolar interval thickness, whereas inflammatory cell infiltration, widened alveolar spaces, and the formation of fibrotic foci were observed in PF patients. As shown in Figure 2E, qRT-PCR indicated that the expression of S100A2 in lung tissues was significantly increased in PF patients, as compared with control groups $(p<0.001)$. Furthermore, immunohistochemistry indicated that the expression of S100A2 was increased in PF tissue (Fig. 2C, D). In addition, we examined S100A2 expression in A549 cells after TGF- $\beta 1$ treatment. As shown in Figure 2F, the mRNA expression of $S 100 A 2$ was significantly increased in TGF- $\beta 1$-stimulated A549 cells. Furthermore, the S100A2 expression in A549 was also evaluated by Western blot analysis (Fig. 2G, H). The S100A2 protein was obviously overexpressed in A549 cells that
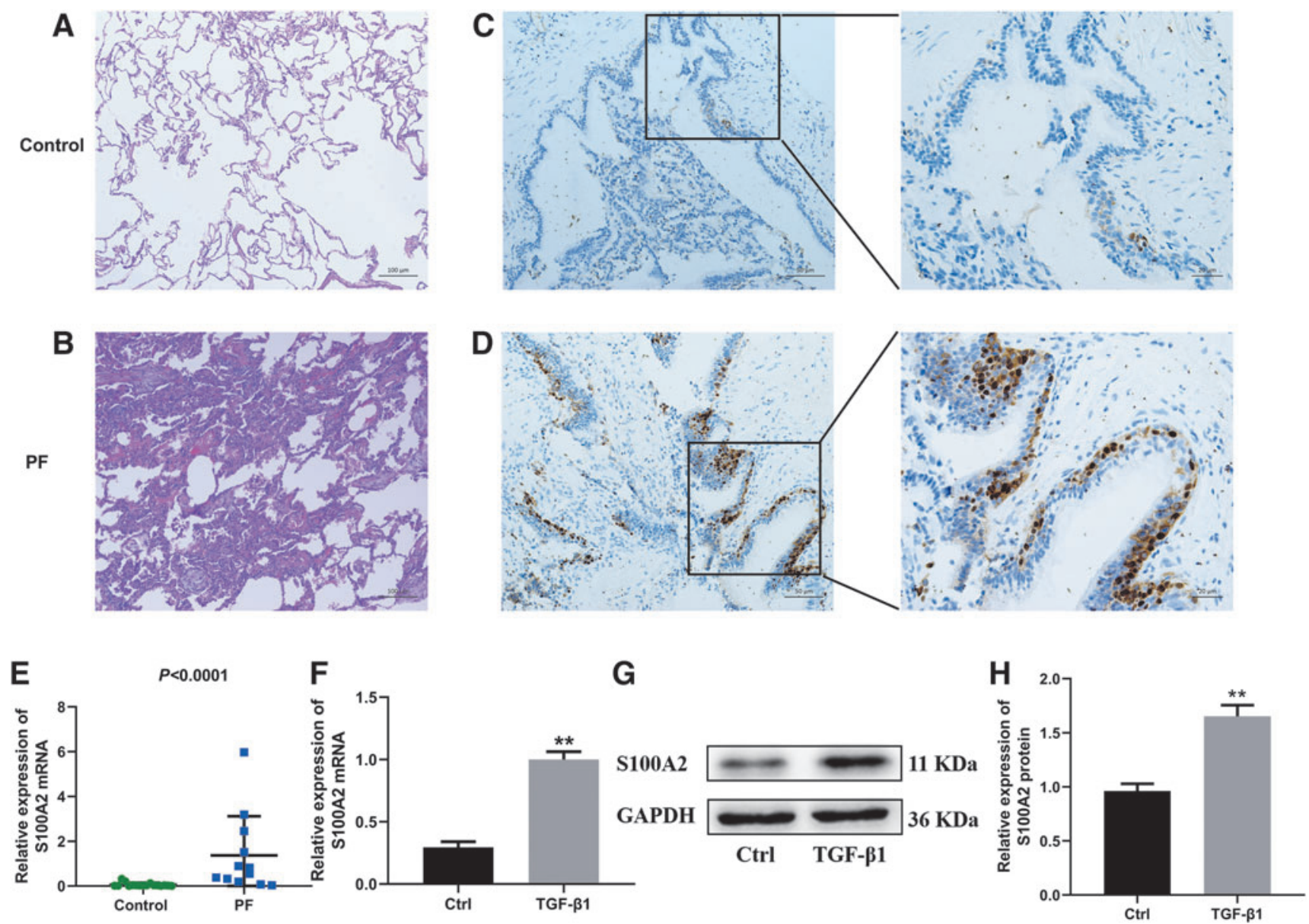

FIG. 2. Expression of $S 100 A 2$ in human PF tissues and human pulmonary epithelial cells (A549) treated with TGF- $\beta 1$. (A, B) Lung tissues were conducted with H\&E staining (original magnification $\times 100$, scale bar: $100 \mu \mathrm{m})$. (C, D) The protein expression of $S 100 A 2$ in human PF tissues and in normal human lung tissues detected by immunohistochemistry (original magnification $\times 200$, scale bar: $50 \mu \mathrm{m}$; original magnification $\times 400$, scale bar: $20 \mu \mathrm{m})$. (E) The mRNA expression of $S 100 A 2$ in human PF tissues and in normal human lung tissues detected by qRT-PCR. A549 cells were treated with or without TGF$\beta 1$ for $48 \mathrm{~h}$. (F) The mRNA expression of S100A2 was determined by qRT-PCR. (G, H) The protein expression of $5100 A 2$ was measured by Western blot. Data are shown as the mean $\pm \mathrm{SD}(n=3)$. $* * p<0.01$ versus Ctrl. Ctrl, control; H\&E, hematoxylin and eosin; qRT-PCR, quantitative real-time PCR; SD, standard deviation; TGF- $\beta 1$, transforming growth factor- $\beta 1$. 
received TGF- $\beta 1$ treatment. Taken together, these findings indicated that $S 100 A 2$ was upregulated both in PF tissues and in A549 cells treated with TGF- $\beta 1$.

\section{Downregulation of S100A2 inhibits TGF- $\beta$ 1-induced EMT in A549 cells}

EMT is characterized by the loss of the epithelial cell marker E-cadherin and overexpression of the mesenchymal markers vimentin and $\alpha$-SMA. TGF- $\beta 1$ is one of the key regulators of $\mathrm{PF}$ and induces EMT of AECs in vitro (Kasai et al., 2005). To investigate the effect of S100A2 on TGF- $\beta 1$ stimulated EMT in A549 cells, S100A2 gene expression knockdown was performed in A549 cells and then stimulated with TGF- $\beta 1$. First, the knockdown efficiency of S100A2 was measured with the use of qRT-PCR and Western blot assay. As shown in Supplementary Figure S1, the mRNA and protein expression of $S 100 A 2$ was dramatically downregulated in the short harpin RNA (shRNA)-S100A2 group compared with the shRNA-negative control group. Downregulation of $S 100 A 2$ obviously increased TGF- $\beta 1$-induced expression of E-cadherin and reduced the expression of vimentin and $\alpha$-SMA in A549 cells both at the mRNA and protein level (Fig. 3A-H), suggesting that S100A2 knockdown inhibited TGF- $\beta 1$-induced EMT.

\section{Deletion of S100A2 suppresses TGF- $\beta 1$-induced EMT in A549 cells through the Wnt/ $\beta$-catenin signaling pathway}

GSK-3 $\beta$ and $\beta$-catenin are two key molecules in the Wnt/ $\beta$-catenin signaling pathway. We used silencing of S100A2
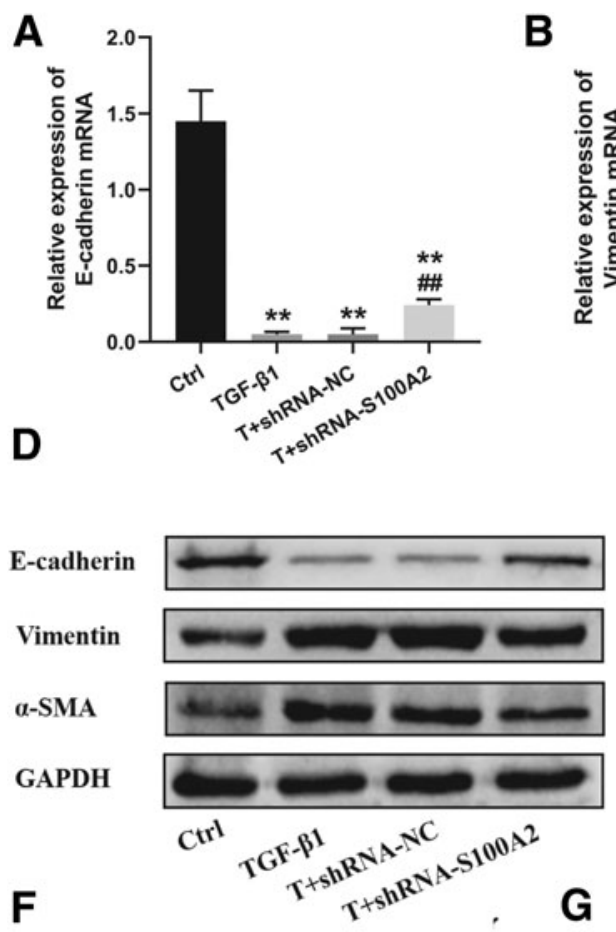

B

G
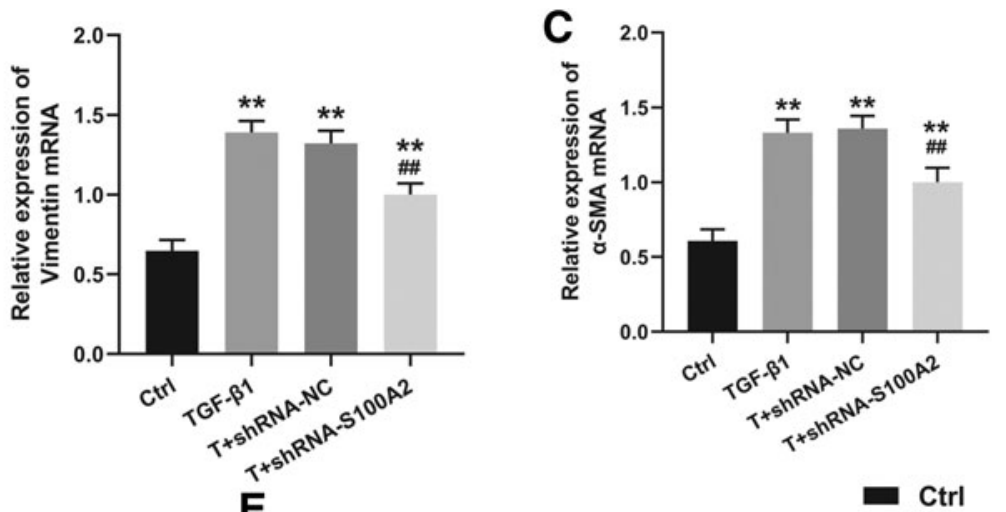

97 KDa

54 KDa

$42 \mathrm{KDa}$

$36 \mathrm{KDa}$
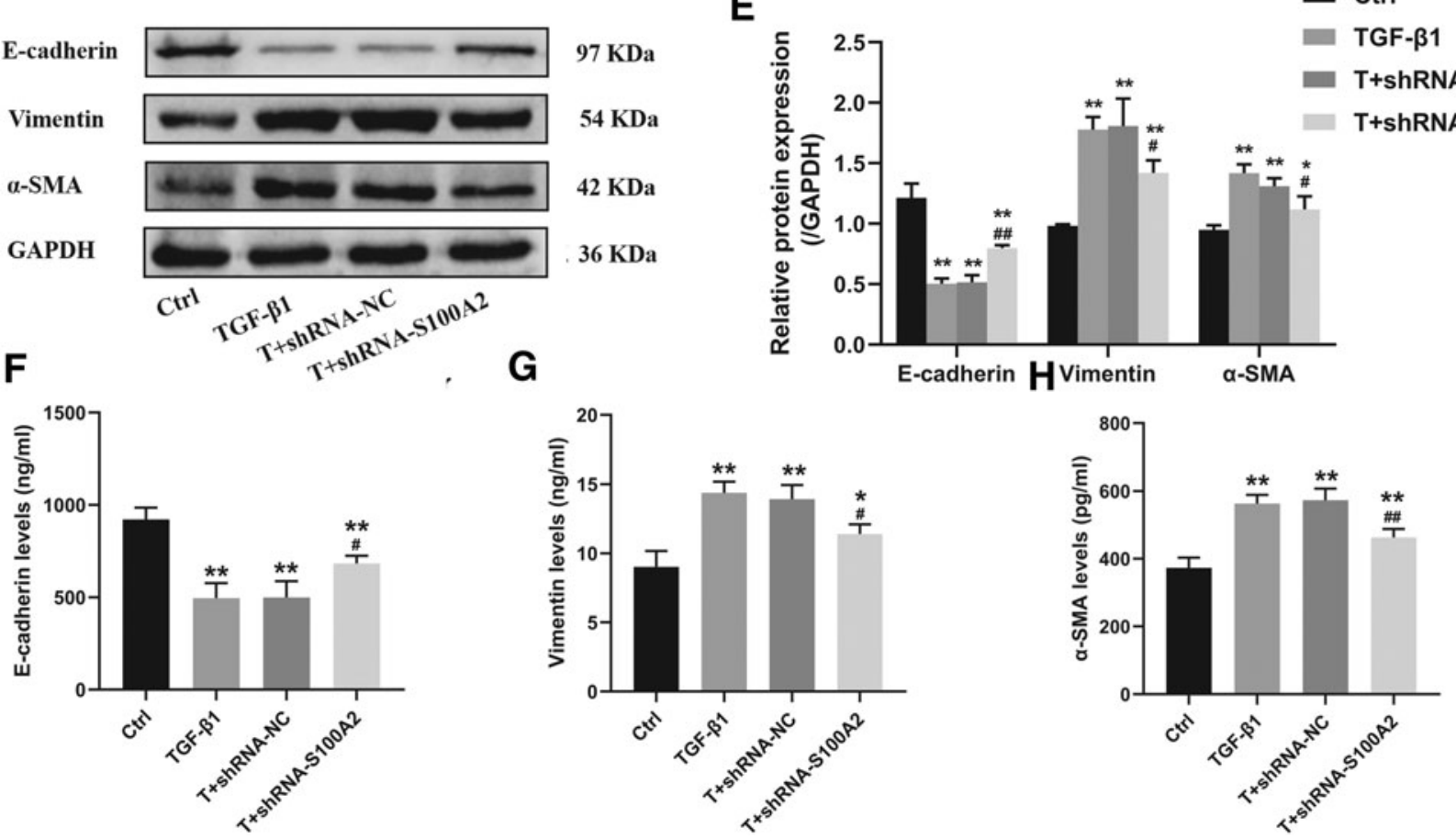

FIG. 3. Effect of SIO0A2 on TGF- $\beta 1$-induced EMT. A549 cells were exposed to $10 \mathrm{ng} / \mathrm{mL}$ TGF- $\beta 1$ stimulation for $48 \mathrm{~h}$. The mRNA of E-cadherin (A), vimentin (B), and $\alpha$-SMA (C) was measured using qRT-PCR. The protein of E-cadherin, vimentin, and $\alpha$-SMA was detected by Western blot (D, E). The concentration of E-cadherin, vimentin, and $\alpha$-SMA in the culture medium without dilution was measured using an enzyme-linked immunosorbent assay kit $(\mathbf{F}-\mathbf{H})$. Data are shown as the mean $\pm \mathrm{SD}(n=3) . * p<0.05$ versus $\mathrm{Ctrl} ; * * p<0.01$ versus Ctrl. ${ }^{*} p<0.05$ versus $\mathrm{T}+$ shRNA-NC; ${ }^{* \#} p<0.01$ versus T+shRNA-NC. $\alpha$-SMA, $\alpha$-smooth muscle actin; EMT, epithelial-mesenchymal transition; shRNA-NC, short harpin RNAnegative control; shRNA-S100A2, short harpin RNA-S100A2; T, TGF- $\beta 1$. 
to determine whether $S 100 A 2$ mediated TGF- $\beta 1$-induced EMT through the Wnt/ $\beta$-catenin pathway. A549 cells were treated with TGF- $\beta 1$, and $\beta$-catenin/GSK- $3 \beta$ protein expression levels were detected using Western blot. Knockdown of S100A2 suppressed TGF- $\beta 1$-induced $\beta$-catenin and phosphorylation of GSK-3 $\beta$, and the protein levels of nonphosphorylated GSK-3 $\beta$ remained unchanged in each group (Fig. 4A-C). These results demonstrated that $S 100 A 2$ blocked TGF- $\beta 1$-induced EMT through downregulation of the $\beta$-catenin pathway.

\section{Reactivation of Wnt/ $\beta$-catenin signaling recovered the suppressive effect of downregulation of S100A2 on EMT}

Finally, we explored whether S100A2 exerts its effect on EMT in a Wnt/ $\beta$-catenin signaling-dependent pathway.
$\mathrm{LiCl}$, which is a $\mathrm{Wnt} / \beta$-catenin signaling pathway activator, was added to A549 cells treated with TGF- $\beta 1$. As shown in Figure $4 \mathrm{D}-\mathrm{H}$, the changes in EMT marker expression (E-cadherin, vimentin, and $\alpha$-SMA) and $\beta$-catenin caused by $S 100 A 2$ downregulation can be reversed by $\mathrm{LiCl}$. Collectively, these findings indicated that downregulation of S100A2 inhibited the EMT of A549 cells by suppressing the $\mathrm{Wnt} / \beta$-catenin signaling pathway.

\section{Discussion}

$\mathrm{PF}$ is characterized by progressive dyspnea, and patients eventually die from respiratory failure. The pathologic mechanisms of PF are not completely clear, and effective therapy has not been well developed. Therefore, it is crucial to explore the precise molecular mechanisms involved in the development of $\mathrm{PF}$ and to determine a target for treatment of PF.
A

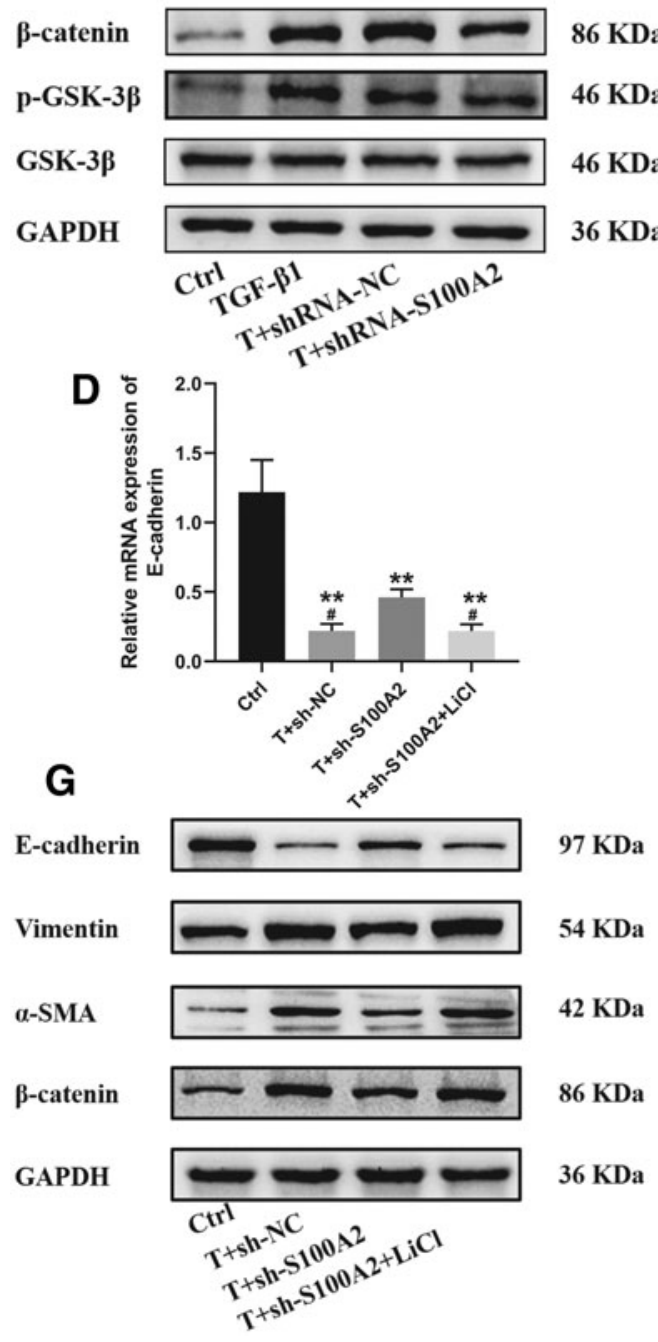

B

$E$
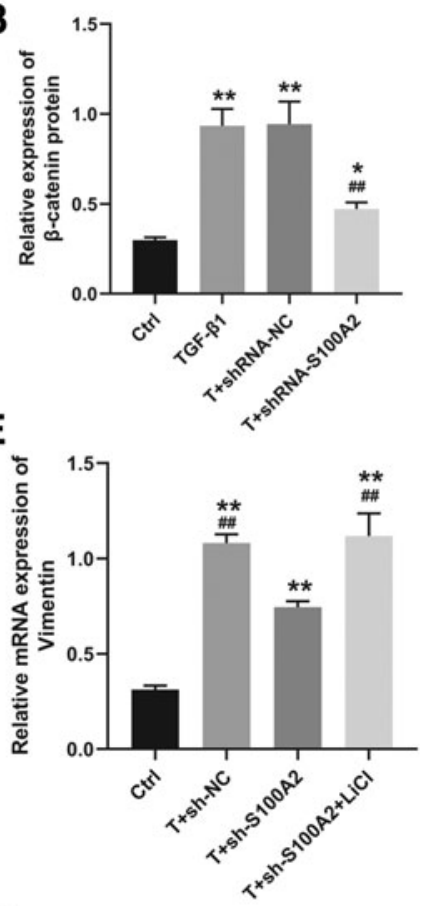

H

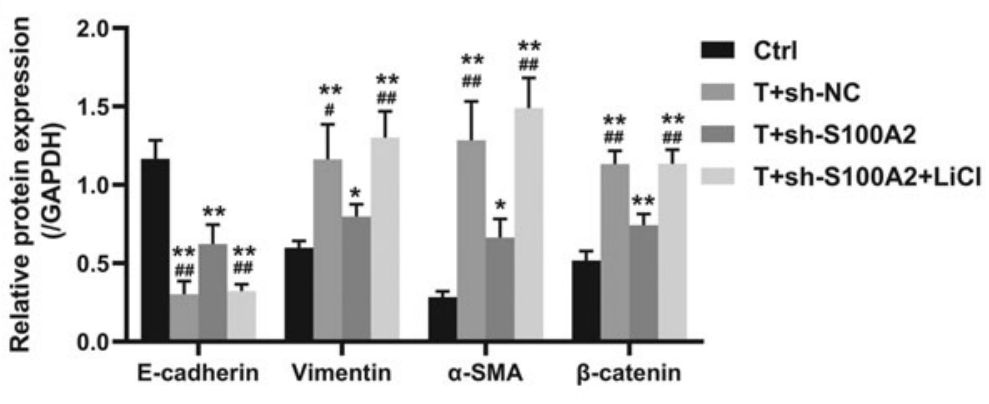

FIG. 4. Downregulation of $S 100 A 2$ suppresses EMT through inhibiting the $\mathrm{Wnt} / \beta$-catenin signaling pathway. The relative protein expression of $\beta$-catenin and p-GSK- $3 \beta$ was determined by Western blot $(\mathbf{A}-\mathbf{C}) .{ }^{*} p<0.05$ versus Ctrl; ${ }^{* *} p<0.01$ versus Ctrl. ${ }^{\#} p<0.05$ versus $\mathrm{T}+$ shRNA-NC; ${ }^{\# \#} p<0.01$ versus $\mathrm{T}+$ shRNA-NC. Reactivation of $\mathrm{Wnt} / \beta$-catenin signaling recovered the suppressive effect of downregulation of $\$ 100 A 2$ on EMT. The relative protein expression of E-cadherin, vimentin, $\alpha$-SMA, and $\beta$-catenin was determined by qRT-PCR (D-F) and Western blot $(\mathbf{G}, \mathbf{H})$. ${ }^{*} p<0.05$ versus Ctrl; ${ }^{* *} p<0.01$ versus Ctrl. ${ }^{\#} p<0.05$ versus $\mathrm{T}+$ sh-S100A2; ${ }^{\# \#} p<0.01$ versus $\mathrm{T}+\mathrm{sh}-\mathrm{S} 100 \mathrm{~A} 2$. Data are shown as the mean $\pm \mathrm{SD}$ $(n=3)$. LiCl, lithium chloride. 
In this study, we explored the expression, regulation, and potential role of $S 100 A 2$ in PF. First, we studied and combined the S100A2 expression of PF patients from the GEO database. The pooled results from the GEO database showed that the expression of $S 100 A 2$ in PF lung tissues was significantly higher than that in normal lung tissues, which demonstrated that $S 100 A 2$ may be involved in PF. Furthermore, we confirmed the increased expression of S100A2 in patients with PF using qRT-PCR and immunohistochemistry assay. Further studies indicated that knockdown of S100A2 suppressed TGF- $\beta 1$-induced EMT in A549 cells. Moreover, our study showed that downregulation of S100A2 inhibited EMT through the $\mathrm{Wnt} / \beta$-catenin pathway. These findings indicated that $S 100 A 2$ was correlated with the development of $\mathrm{PF}$ and may serve as a new target for the prevention and treatment of $\mathrm{PF}$.

S100A2 is widely studied in various cancers. Many studies indicate that there is a certain similarity between tumor metastasis and fibrotic diseases. For instance, the dysregulated TGF- $\beta 1$ pathway and activated EMT process are involved in these two diseases. Downregulation of S100A2 was detected in gastric cancer and oral squamous cell cancer (Wolf et al., 2011). However, increased S100A2 expression was observed in ovarian cancer (Hough et al., 2001), bladder cancer (Yao et al., 2007), and nonsmall cell lung cancer (Bulk et al., 2009). In this study, our data showed the first evidence that the expression of $5100 A 2$ was increased in PF lung tissue.

EMT is involved in the pathogenesis of fibrotic diseases in many organs, including the lung. TGF- $\beta 1$ is considered to be a key profibrotic cytokine and a regulator of EMT. It is well established that AECs, particularly type II AECs, obtain their phenotype of fibroblasts through EMT to contribute to the development of PF (Willis et al., 2005; Kim et al., 2006). Although A549 cell is a cancer cell line, the cells retain the characteristics of type II AECs (Foster et al., 1998; Wu et al., 2017). In addition, AECs are difficult to obtain and maintain in culture ex vivo. Therefore, the A549 cell line is usually used as a replacement for primary AECs and is extensively used to study the mechanism of PF (Uhal et al., 2013; Zhu et al., 2016; Liu et al., 2019). In this study, we employed TGF- $\beta 1$ to build an EMT model using the A549 cell line. In our study, we demonstrated that downregulation of S100A2 inhibited TGF- $\beta 1$-induced EMT.

The Wnt/ $\beta$-catenin signaling pathway plays essential roles in a variety of biological processes, including embryonic axial development, organogenesis, adult stem cell maintenance, and tissue homeostasis (Moon et al., 2004; Brack et al., 2007; Clevers and Nusse, 2012). The key molecule of the pathway is $\beta$-catenin. Under normal conditions, $\beta$-catenin is phosphorylated by a destruction complex that is composed of axin, GSK-3 $\beta$, and adenomatous polyposis coli protein, and then ubiquitinated and ultimately destroyed by the proteasome (Guo et al., 2012). Under pathological conditions, Wnt proteins bind to their receptors, and result in the disassembly of the destruction complex, leading to dephosphorylation of $\beta$-catenin. Subsequently, $\beta$-catenin is allowed to translocate into the nucleus, where it binds to T cell factor/ lymphoid enhancer factor, which is in the transcription factor family, to trigger the transcription of $\beta$-catenin target genes (Rao and Kühl, 2010). It has been demonstrated that the
Wnt/ $\beta$-catenin signaling pathway plays an important role in the pathological processes involving $\mathrm{PF}$, and suppression of $\mathrm{Wnt} / \beta$-catenin signaling could inhibit the development of PF (Chilosi et al., 2003; Wang et al., 2014, 2015). GSK-3 $\beta$ is a serine/threonine protein kinase that promotes fibrogenic activity by participating in the execution of $\mathrm{Wnt} / \beta$-catenin pathway (Beurel et al., 2015; Singh et al., 2015; Boyapally et al., 2019). In this study, we discovered that inhibition of $S 100 A 2$ restrains EMT by inhibiting the $\mathrm{Wnt} / \beta$-catenin pathway.

In conclusion, we demonstrated that the expression of $S 100 A 2$ was increased in the lung tissues in patients with $\mathrm{PF}$. Inhibition of $S 100 A 2$ can attenuate TGF- $\beta 1$-induced EMT through inhibiting the $\beta$-catenin signaling pathway. $S 100 A 2$ is a promising potential target for further understanding the mechanism and developing a strategy for the treatment of PF and other EMT-associated diseases.

\section{Disclosure Statement}

The authors declare that they have no conflict of interest.

\section{Funding Information}

This research was supported by the National Major Science and Technology Projects of China (Grant No. 2018ZX10 302302003).

\section{Supplementary Material}

Supplementary Figure S1

Supplementary Table S1

Supplementary Table S2

\section{References}

Beurel, E., Grieco, S.F., and Jope, R.S. (2015). Glycogen synthase kinase-3 (GSK3): regulation, actions, and diseases. Pharmacol Ther 148, 114-131.

Boyapally, R., Pulivendala, G., Bale, S., and Godugu, C. (2019). Niclosamide alleviates pulmonary fibrosis in vitro and in vivo by attenuation of epithelial-to-mesenchymal transition, matrix proteins \& Wnt/beta-catenin signaling: a drug repurposing study. Life Sci 220, 8-20.

Brack, A.S., Conboy, M.J., Roy, S., Lee, M., Kuo, C.J., Keller, C., et al. (2007). Increased Wnt signaling during aging alters muscle stem cell fate and increases fibrosis. Science $\mathbf{3 1 7}$, 807-810.

Bulk, E., Sargin, B., Krug, U., Hascher, A., Jun, Y., Knop, M., et al. (2009). S100A2 induces metastasis in non-small cell lung cancer. Clin Cancer Res 15, 22-29.

Chen, H., Xu, C., Jin, Q., and Liu, Z. (2014). S100 protein family in human cancer. Am J Cancer Res 4, 89-115.

Chilosi, M., Poletti, V., Zamo, A., Lestani, M., Montagna, L., Piccoli, P., et al. (2003). Aberrant Wnt/beta-catenin pathway activation in idiopathic pulmonary fibrosis. Am J Pathol 162, 1495-1502.

Clevers, H., and Nusse, R. (2012). Wnt/ $\beta$-catenin signaling and disease. Cell 149, 1192-1205.

Du, S., Li, C., Lu, Y., Lei, X., Zhang, Y., Li, S., et al. (2019). Dioscin alleviates crystalline silica-induced pulmonary inflammation and fibrosis through promoting alveolar macrophage autophagy. Theranostics 9, 1878-1892.

Foster, K.A., Oster, C.G., Mayer, M.M., Avery, M.L., and Audus, K.L. (1998). Characterization of the A549 cell line as 
a type II pulmonary epithelial cell model for drug metabolism. Exp Cell Res 243, 359-366.

George, P.M., Wells, A.U., and Jenkins, R.G. (2020). Pulmonary fibrosis and COVID-19: the potential role for antifibrotic therapy. Lancet Respir Med 8, 807-815.

Guo, Y., Xiao, L., Sun, L., and Liu, F. (2012). Wnt/beta-catenin signaling: a promising new target for fibrosis diseases. Physiol Res 61, 337-346.

He, F., Wang, Y., Li, Y., and Yu, L. (2020). Human amniotic mesenchymal stem cells alleviate paraquat-induced pulmonary fibrosis in rats by inhibiting the inflammatory response. Life Sci 243, 117290.

Hough, C.D., Cho, K.R., Zonderman, A.B., Schwartz, D.R., and Morin, P.J. (2001). Coordinately up-regulated genes in ovarian cancer. Cancer Res 61, 3869-3876.

Jolly, M.K., Ward, C., Eapen, M.S., Myers, S., Hallgren, O., Levine, H., et al. (2018). Epithelial-mesenchymal transition, a spectrum of states: role in lung development, homeostasis, and disease. Dev Dyn 247, 346-358.

Kasai, H., Allen, J.T., Mason, R.M., Kamimura, T., and Zhang, Z. (2005). TGF-beta1 induces human alveolar epithelial to mesenchymal cell transition (EMT). Respir Res 6, 56.

Kim, K.K., Kugler, M.C., Wolters, P.J., Robillard, L., Galvez, M.G., Brumwell, A.N., et al. (2006). Alveolar epithelial cell mesenchymal transition develops in vivo during pulmonary fibrosis and is regulated by the extracellular matrix. Proc Natl Acad Sci U S A 103, 13180-13185.

Kumar, A., Kapnadak, S.G., Girgis, R.E., and Raghu, G. (2018). Lung transplantation in idiopathic pulmonary fibrosis. Expert Rev Respir Med 12, 375-385.

Liu, G., Wang, Y., Yang, L., Zou, B., Gao, S., Song, Z., et al. (2019). Tetraspanin 1 as a mediator of fibrosis inhibits EMT process and Smad2/3 and beta-catenin pathway in human pulmonary fibrosis. J Cell Mol Med 23, 3583-3596.

Moon, R.T., Kohn, A.D., De-Ferrari G.V., and Kaykas, A. (2004). WNT and beta-catenin signalling: diseases and therapies. Nat Rev Genet 5, 691-701.

Naz, S., Bashir, M., Ranganathan, P., Bodapati, P., Santosh, V., and Kondaiah, P. (2014). Protumorigenic actions of S100A2 involve regulation of PI3/Akt signaling and functional interaction with Smad3. Carcinogenesis 35, 14-23.

Pan, S.C., Li, C.Y., Kuo, C.Y., Kuo, Y.Z., Fang, W.Y., Huang, Y.H., et al. (2018). The p53-S100A2 positive feedback loop negatively regulates epithelialization in cutaneous wound healing. Sci Rep 8, 5458.

Rao, T.P., and Kühl, M. (2010). An updated overview on Wnt signaling pathways: a prelude for more. Circ Res 106, 17981806.

Salton, F., Volpe, M.C., and Confalonieri, M. (2019). Epithelialmesenchymal transition in the pathogenesis of idiopathic pulmonary fibrosis. Medicina (Kaunas) 55, 83.

Singh, S.P., Tao, S., Fields, T.A., Webb, S., Harris, R.C., and Rao, R. (2015). Glycogen synthase kinase-3 inhibition at- tenuates fibroblast activation and development of fibrosis following renal ischemia-reperfusion in mice. Dis Model Mech 8, 931-940.

Uhal, B.D., Dang, M., Dang, V., Llatos, R., Cano, E., AbdulHafez, A., et al. (2013). Cell cycle dependence of ACE-2 explains downregulation in idiopathic pulmonary fibrosis. Eur Respir J 42, 198-210.

Wang, C., Zhu, H., Sun, Z., Xiang, Z., Ge, Y., Ni, C., et al. (2014). Inhibition of $\mathrm{Wnt} /$ beta-catenin signaling promotes epithelial differentiation of mesenchymal stem cells and repairs bleomycin-induced lung injury. Am J Physiol Cell Physiol 307, C234-C244.

Wang, X., Dai, W., Wang, Y., Gu, Q., Yang, D., and Zhang, M. (2015). Blocking the Wnt/beta-catenin pathway by lentivirusmediated short hairpin RNA targeting beta-catenin gene suppresses silica-induced lung fibrosis in mice. Int J Environ Res Public Health 12, 10739-10754.

Willis, B.C., and Borok, Z. (2007). TGF-beta-induced EMT: mechanisms and implications for fibrotic lung disease. Am J Physiol Lung Cell Mol Physiol 293, L525-L534.

Willis, B.C., Liebler, J.M., Luby-Phelps, K., Nicholson, A.G., Crandall, E.D., du Bois, R.M., et al. (2005). Induction of epithelial-mesenchymal transition in alveolar epithelial cells by transforming growth factor-beta1: potential role in idiopathic pulmonary fibrosis. Am J Pathol 166, 1321-1332.

Wolf, S., Haase-Kohn, C., and Pietzsch, J. (2011). S100A2 in cancerogenesis: a friend or a foe? Amino Acids 41, 849-861.

Wu, J., Wang, Y., Liu, G., Jia, Y., Yang, J., Shi, J., et al. (2017). Characterization of air-liquid interface culture of A549 alveolar epithelial cells. Braz J Med Biol Res 51:e6950.

Yao, R., Lopez-Beltran, A., Maclennan, G.T., Montironi, R., Eble, J.N., and Cheng, L. (2007). Expression of S100 protein family members in the pathogenesis of bladder tumors. Anticancer Res 27, 3051-3058.

Zhu, Y., Tan, J., Xie, H., Wang, J., Meng, X., and Wang, R. (2016). HIF- $1 \alpha$ regulates EMT via the Snail and $\beta$-catenin pathways in paraquat poisoning-induced early pulmonary fibrosis. J Cell Mol Med 20, 688-697.

Address correspondence to: Shuliang Guo, PhD

Department of Pulmonary and Critical Care Medicine The First Affiliated Hospital of Chongqing Medical University

No. 1, Youyi Road

Yuzhong, Chongqing 400016

China

E-mail: guos1999@sina.com

Received for publication August 10, 2020; received in revised form September 25, 2020; accepted October 9, 2020. 